\title{
Curve Following for Nonlinear Dynamic Systems Subject to Control Constraints
}

\author{
Mats Jirstrand \\ Department of Electrical Engineering \\ Linköping University, S-581 83 Linköping, Sweden \\ www: http://www. control.isy.liu.se \\ email: matsj@isy.liu.se \\ 1997-04-07
}

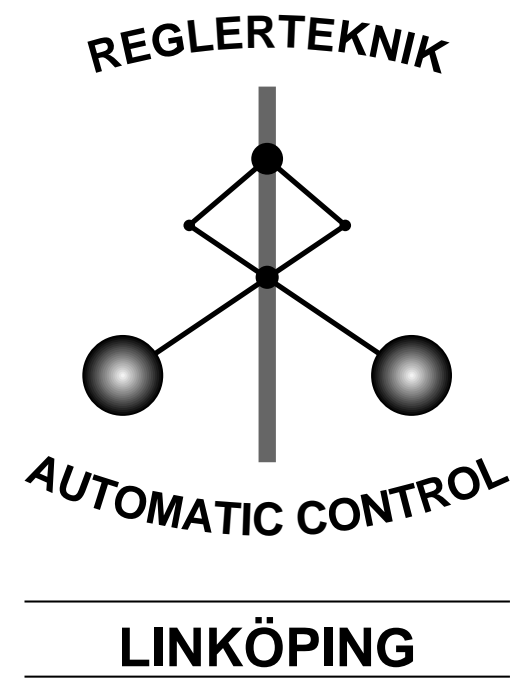

Technical reports from the Automatic Control group in Linköping are available as UNIX-compressed Postscript files by anonymous ftp at the address 130.236.20.24 (ftp.control.isy.liu.se). 


\title{
Curve Following for Nonlinear Dynamic Systems Subject to Control Constraints
}

\author{
Mats Jirstrand \\ Department of Electrical Engineering, \\ Linköping University, 58183 Linköping, Sweden \\ www: http://www.control.isy.liu.se \\ email: matsj@isy.liu.se
}

\begin{abstract}
A constructive method for investigating if a given nonlinear dynamic system subject to control constraints can be controlled to follow a desired curve in the state or output space is presented. If the method gives an affirmative answer we can compute a closed form expression of the corresponding state feedback control law. Specifying a family of parametrized curves, approximations of reachable sets can also be computed.
\end{abstract}

Keywords: curve following, tracking, quantifier elimination, nonlinear systems, control constraints, reachability

\section{Introduction}

In many control applications one of the main objectives is to track or follow a given trajectory in the state or output space of a dynamic system. There are several approaches to control law design for such problems, e.g., adaptive control or local control laws based on linearizations of the system around the trajectory. However, these methods does not take control constraints, such as amplitude and rate limitations, into account. Usually one has to verify the design afterwards by simulations to check that the constraints are not violated. For nonlinear systems it is usually very hard to show that the simulations have covered all possibilities. Hence, there is a need for more systematic procedures to verify that a given system can follow a prescribed trajectory in the state-space using only admissible values of the control signals.

A slight variation of the curve following problem can be used to compute approximations of the set of states that is reachable from a given set of possible initial states. Not only control constraints but also constraints on the states can be taken into account.

In this paper we address the above problems in an algebraic framework using a constructive algebraic method known as quantifier elimination. Software for symbolic computations such as MATHEMATICA and QEPCAD plays a crucial role since the algebraic manipulations soon becomes impossible to do by hand even for small problems.

The problems in this paper can be formulated in terms of polynomial equations, inequalities, quantifiers $(\exists, \forall)$, and Boolean operators $(\rightarrow, \vee, \wedge)$, which we call formulas. A quantifier elimination algorithm can then be used to decide if the formula is True or FAlse, or to derive algebraic constraints on design and controller parameters, such that a control law for the specified curve exists.

Introductions to quantifier elimination can be found in, $[12,7]$. For an extensive bibliography on the subject, see [3]. In control theory, one of the first attempts to use quantifier elimination techniques was made by Anderson et al. [2] and recently, a few papers treating control related problems have appeared $[1,8,9,10,13]$.

In Section 2 we formulate the curve following problem as a formula including quantified variables and show how quantifier elimination can be used to decide if the problem is solvable. Section 3 treats the question how to design a state feedback control law. Approximations of reachable sets is presented in Section 4. A summary of the paper and some conclusions is given in Section 5.

\section{Curve Following}

Consider a dynamic system described by a nonlinear differential equation written on state space form

$$
\begin{aligned}
& \dot{x}=f(x, u) \\
& y=h(x)
\end{aligned}
$$

where $x \in \mathbb{R}^{n}, u \in \mathbb{R}^{m}, y \in \mathbb{R}^{p}$ and each component of $f$ and $h$ is a real polynomial, $f_{i} \in \mathbb{R}[x, u], h_{j} \in \mathbb{R}[x]$. The $x, u$, and $y$ vectors will be referred to as the state, control, and output of the system, respectively.

The restriction to polynomial systems is not very severe. It can be shown that systems where the nonlinearities are not originally polynomial may be rewritten on polynomial form if the nonlinearities themselves are solutions to algebraic differential equations. For more details on this the reader is referred to $[14,11]$.

To describe constraints on variables we use semialgebraic sets formally defined as follows.

Definition 1 A subset of $\mathbb{R}^{n}$ is semi-algebraic if it can be constructed by finitely many applications of union, intersection and complementation operations on sets of the 


$$
\left\{x \in \mathbb{R}^{n} \mid f(x) \geq 0\right\},
$$

where $f \in \mathbb{R}\left[x_{1}, \ldots, x_{n}\right]$.

Restrictions on amplitudes of control signals are common in real applications but hard to take into account in many classical design methods other than by simulation studies. We assume that the control of system (1) has to obey some additional constraints $u \in \mathcal{U}$, where $\mathcal{U}$ is a semialgebraic set. We call $u \in \mathcal{U}$ the admissible controls. A variety of constraints can be represented by semi-algebraic sets, e.g., amplitude and direction constraints.

Example 1 Let $\mathbf{F}$ be a two dimensional thrust vector which can be pointed in any direction, $\phi$ and whose magnitude, $|\mathbf{F}|$ can be varied between 0 and $F_{\text {max }}$. Let $u_{1}=\cos (\phi), \quad u_{2}=\sin (\phi) \quad$ and $\quad u_{3}=|\mathbf{F}|$. Then a semialgebraic set describing these constraints are

$$
\mathcal{U}=\left\{u \in \mathbb{R}^{3} \mid u_{1}^{2}+u_{2}^{2}=1 \wedge 0 \leq u_{3} \leq F_{\max }\right\}
$$

Consider system (1) subject to some semi-algebraic control constraints, $u \in \mathcal{U}$. Let $\Gamma$ be a rationally parametrized curve in the output space $\mathbb{R}^{p}$, i.e.,

$$
\Gamma=\left\{y \in \mathbb{R}^{p} \mid y=g(s), g: \mathbb{R} \rightarrow \mathbb{R}^{p}, s \in[\alpha, \beta]\right\},
$$

where the orientation of $\Gamma$ is defined by increasing values of $s$.

To steer the system along a curve in the state space there has to be an admissible control $u$ at each point on the curve such that the solution trajectory tangent vector $f(x, u)$ points in the same direction as a forward pointing tangent vector of the curve, i.e.,

$$
f(g(s), u)=\lambda \frac{\mathrm{d}}{\mathrm{ds}} g(s), \quad \lambda>0, \quad \forall s \in[\alpha, \beta] .
$$

The more general case when a curve in the output space is given is treated in the following theorem. Note that if the number of outputs is less than the number of states, a curve in the output space corresponds to a manifold of dimension $>1$ in the state space.

Theorem 1 There exists an admissible control $u \in \mathcal{U}$ such that the output $y$ of system (1) follows the curve $\Gamma$ iff the formula

$$
\begin{aligned}
& (\forall s \in[\alpha, \beta])(\exists x)(\exists u \in \mathcal{U})(\exists \lambda>0) \\
& {\left[h_{x}(x) f(x, u)=\lambda \frac{\mathrm{d}}{\mathrm{ds}} g(s) \wedge g(s)=h(x)\right]}
\end{aligned}
$$

is TRue.
Proof: The curve $\Gamma$ in the output space can be followed iff the output trajectory tangent $\dot{y}(t)$ can be chosen parallel to the tangent vector of $\Gamma$, at each point on $\Gamma$, by an admissible choice of $u$. Now

$$
\dot{y}(t)=h_{x}(x) \dot{x}=h_{x}(x) f(x, u)
$$

and a tangent of $\Gamma$ is given by $\frac{\mathrm{d}}{\mathrm{ds}} g(s)$. Hence, the parallel condition becomes

$$
h_{x}(x) f(x, u)=\lambda \frac{\mathrm{d}}{\mathrm{ds}} g(s)
$$

for some $\lambda>0, u \in \mathcal{U}$ and the theorem follows.

Now, quantifier elimination can be used to eliminate all quantified variables. In (3) all variables are quantified and the quantifier elimination algorithm gives TRUE or FALSE as a result, i.e., either there is an admissible control that steers the system a long the curve or not. If the equations that specify the dynamic system or the curve contain additional parameters, then the quantifier elimination gives necessary and sufficient conditions on these free parameters such that an admissible control law exists. Solutions to these systems of polynomial equations and inequalities can, e.g., be computed by cylindrical algebraic decomposition [4, 5, 12], which in many cases also is a part of the quantifier elimination algorithm. Hence a feasible solution is often produced directly.

Example 2 Consider the following system

$$
\begin{aligned}
\dot{x}_{1} & =-a x_{2}+\frac{1}{2} x_{1} x_{2}, \\
\dot{x}_{2} & =-x_{1}-x_{2}+\left(1+x_{1}^{2}\right) u, \\
y & =\left(x_{1}, x_{2}\right)^{T}
\end{aligned}
$$

where $-2 \leq u \leq 2$ and $\Gamma$ is the unit circle. Here we have one free design parameter $a$. The unit circle can be rationally parametrized according to

$$
\Gamma=\left\{x \in \mathbb{R}^{2} \mid x_{1}=\frac{s^{2}-1}{s^{2}+1}, x_{2}=\frac{-2 s}{s^{2}+1}, s \in \mathbb{R}\right\} .
$$

In this example the output map is the identity. Hence we can eliminate $x$ directly by substitution. Clearing denominators the parallel condition in formula (3) becomes

$$
\begin{aligned}
& s+2 b s-s^{3}+2 b s^{3}=4 \lambda s \wedge \\
& 1+2 s+2 s^{3}-s^{4}+2 u+2 s^{4} u=2 \lambda\left(s^{2}-1\right)
\end{aligned}
$$


Next follows a few lines of Mathematica code:

$$
\begin{aligned}
\text { In [1] : }= & \text { EliminateQuantifiers }[ \\
& \forall_{s} \exists_{u} \exists_{\lambda} \\
& \left(s+2 a s-s^{3}+2 a s^{3}=4 \lambda s \& \&\right. \\
& 1+2 s+2 s^{3}-s^{4}+2 u+2 s^{4} u=2 \lambda\left(s^{2}-1\right) \\
& \& \& \quad \lambda>0 \& \&-2 \leq u \text { \&\& } u \leq 2)] \\
\text { Out [1] }= & -1+2 a \geq 0 \& \&-4409+2548 a+790 a^{2}- \\
& 452 a^{3}-73 a^{4}+24 a^{5}+4 a^{6} \leq 0 \\
\text { In }[2]:= & \mathrm{N}[\text { InequalitySolve }[\%, \text { a }]] \\
\text { Out [2] }= & 0.5 \leq a \leq 1.8819
\end{aligned}
$$

Here the function EliminateQuantifiers calls the external program QEPCAD and returns the output from this program. The second line of input is a built-in MATHEMATICA function for simplification of Boolean combinations of univariate inequalities.

Hence, there is an admissible control such that the output (states) of system (4) follows the unit circle if and only if $\frac{1}{2} \leq a \leq \alpha$, where $\alpha \approx 1.8819$ is given by a real zero of a sixth order polynomial.

\section{Control Law Design}

How do we construct a control law that steers the system along the curve $\Gamma$ once the quantifier elimination in formula (3) gives an affirmative answer?

\subsection{Open Loop Control Law}

To construct an open loop control law, i.e., a control law of the form $\mathrm{u}=\mathrm{u}(\mathrm{t})$, where $\mathrm{u}(\mathrm{t})$ is a given function of time we only have to eliminate quantifiers in

$$
\begin{aligned}
(\exists \lambda>0) & (\exists x) \\
& {\left[h_{x}(x) f(x, u)=\lambda \frac{\mathrm{d}}{\mathrm{ds}} g(s) \wedge g(s)=h(x)\right] . }
\end{aligned}
$$

The result is an implicit expression in $u$ and $s$. Solving for $u$ in this expression gives an open loop control law, where $s$ corresponds to time. A time scaling $s=\varphi(t)$, where $\varphi(\cdot)$ is a monotonically increasing function can then be used to gain some additional freedom without affecting the alignments of $h_{x}(x) f(x, u)$ and $\frac{\mathrm{d}}{\mathrm{ds}} g(s)$. However, after this operation we have to check that $u$ is still admissible.

\subsection{State Feedback Control Law}

A state feedback control law can be computed by first eliminating quantifiers in

$$
\begin{aligned}
& (\exists \lambda>0)(\exists s \in[\alpha, \beta]) \\
& \quad\left[h_{x}(x) f(x, u)=\lambda \frac{\mathrm{d}}{\mathrm{ds}} g(s) \wedge g(s)=h(x)\right],
\end{aligned}
$$

which gives the implicit form of an state feedback control law. However, for the computation of a state feedback law the use of quantifier elimination algorithms leads to unnecessarily complex computations. Next we describe an alternatively approach.

We start with computing the implicit form of the set in the state space corresponding to the parametrized curve $\Gamma$ in the output space, i.e., eliminating $s$ from $g(s)=h(x)$. This implicitization problem is easily solved using for example Gröbner bases, see [6]. If the number of outputs $p$ is less than the number of states $n$ the implicitization procedure gives an implicit description of a manifold $\mathcal{C}$ in the state space (algebraic variety of dimension $>1$ ) whose map under $h(x)$ include $\Gamma$ as a subset.

Now, let the equations describing the manifold $\mathcal{C}$ be denoted by $c_{i}(x)=0, i=1, \ldots, s$ or by vector notation

$$
c(x)=0
$$

The state trajectory tangent, $f(x, u)$ has to be an element of the tangent space of $\mathcal{C}$ at each point on the curve for the output to remain on $\Gamma$. This condition can be formulated as follows

$$
c_{x}(x) f(x, u)=0,
$$

which is an implicit expression for the desired state feedback control law and we have to solve for $u$ to get the final form.

However, this is not the most general implicit form of an algebraic state feedback control law. Since the polynomials $c_{i}(x)$ is zero on $\mathcal{C}$ a more general implicit control law is given by

$$
\begin{aligned}
& c_{x}(x) f(x, u)=v, \\
& v_{j}=\sum_{i=1}^{s} q_{i j}(x) c_{i}(x), q_{i j} \in \mathbb{R}[x] .
\end{aligned}
$$

These control laws give identical system behavior on $\mathcal{C}$ but the extra freedom (free choice of the polynomials $q_{i j}(x)$ ) can be used to tune the system behavior in $\mathbb{R}^{n} \backslash \mathcal{C}$.

Example 3 Consider system (4) again. The implicit description of curve $\mathcal{C}$ can be computed as outlined above. However in this simple case we already know the implicit form of $\mathcal{C}: x_{1}^{2}+x_{2}^{2}-1=0$. The orthogonality condition (8) becomes

$$
\begin{aligned}
x_{1}\left(-a x_{2}+\frac{1}{2} x_{1} x_{2}\right) & =q_{1}(x)\left(x_{1}^{2}+x_{2}^{2}-1\right) \\
x_{2}\left(-x_{1}-x_{2}+\left(1+x_{1}^{2}\right) u\right) & =q_{2}(x)\left(x_{1}^{2}+x_{2}^{2}-1\right)
\end{aligned}
$$

where $q_{1}(x), q_{2}(x) \in \mathbb{R}[x]$. Here the first equation does not impose any constraints on $u$ and solving for $u$ in the second equation gives

$$
u=-\frac{(2+2 a) x_{1}+2 x_{2}-x_{1}^{2}}{2\left(1+x_{1}^{2}\right)} .
$$

A simulation of the closed loop system behavior for $q_{1}=$ $q_{2}=0, a=0.55$, and $a=1.88$, respectively, is shown in Figure 1. 

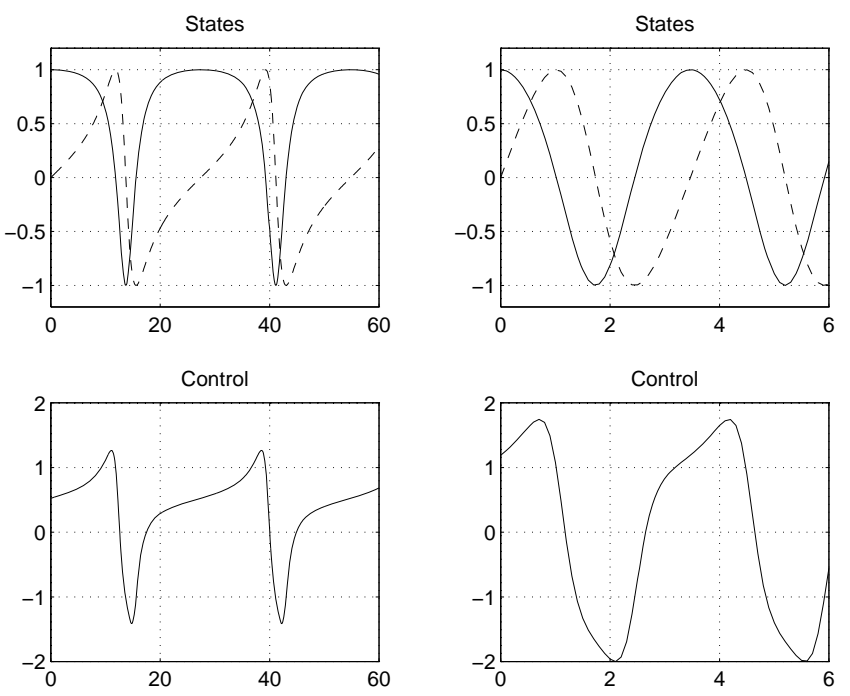

Figure 1: The states and control behavior for $a=0.55$ (left) and $a=1.88$ (right), respectively.

The computed control law does not force the state to converge to $\Gamma$ if the initial state is outside the curve. However, one can show that using the modified control law

$$
u=-\frac{(2+2 a) x_{1}+2 x_{2}-x_{1}^{2}}{2\left(1+x_{1}^{2}\right)}-2 x_{2}\left(x_{1}^{2}+x_{2}^{2}-1\right),
$$

convergence for initial conditions in a neighborhood of $\Gamma$ is obtained. In Figure 2 a simulation of system (4) for $a=1$ and different initial conditions is shown.

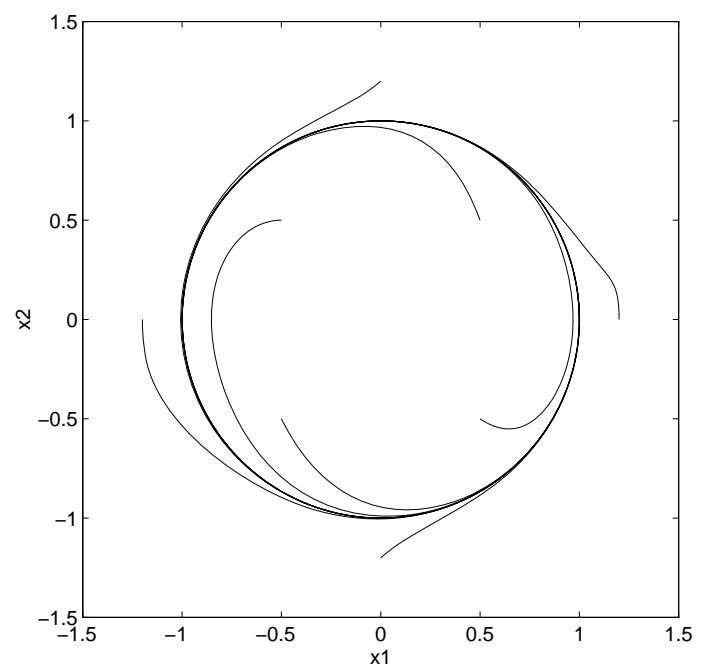

Figure 2: A phase portrait of system (4) when the modified control law is used.
Observe that the symbolic nature of the quantifier elimination method makes it possible to have symbolic parameters undetermined during the whole computation, from system description (1) to the final expression of the control law. Hence the control law is valid for all parameters satisfying the algebraic constraints that results from quantifier elimination in (3).

\section{Reachability}

The ideas on curve following can be generalized to give approximations of the set of reachable states of system (1) subject to some control constraints. Let $\mathcal{X}_{0}$ be a given semi-algebraic set of initial states and $\mathcal{G}$ a family of rationally parametrized curves

$$
\begin{aligned}
\mathcal{G}=\left\{x \in \mathbb{R}^{n} \mid\right. & x=g\left(s ; x^{0}, x^{1}, \theta\right), s \in[0,1], \\
& \left.x^{0} \in \mathcal{X}_{0}, \theta \in \Theta, g\left(1 ; x^{0}, x^{1}, \theta\right)=x^{1}\right\},
\end{aligned}
$$

where $\Theta$ is a semi-algebraic set of shape parameters.

Definition 2 A point $x^{1}$ in the state space of system (1) is $\mathcal{G}$-reachable from $\mathcal{X}_{0}$ if there is a curve $\Gamma \in \mathcal{G}$ such that $x^{1}$ is reachable along $\Gamma$ using an admissible control.

We can formulate those states that are reachable from $\mathcal{X}_{0}$ along one of the curves in $\mathcal{G}$ as a formula including quantifiers similarly to formula (3).

Theorem 2 The $\mathcal{G}$-reachable states of system (1) is given by the following formula

$$
\begin{aligned}
(\exists \theta \in \Theta) & \left(\exists x_{0} \in \mathcal{X}_{0}\right)(\forall s \in[0,1]) \\
& (\exists u \in \mathcal{U})(\exists \lambda>0)\left[f(g, u)=\lambda \frac{\mathrm{d}}{\mathrm{ds}} g\right],
\end{aligned}
$$

where $g \in \mathcal{G}$.

Proof: Similar to the proof of Theorem 1.

Performing quantifier elimination gives a formula in $x^{1}$ describing the states that can be reached along at least one of the curves in $\mathcal{G}$ using an admissible control.

The first two quantifiers select a curve in $\mathcal{G}$, the other quantifiers is similar to the ones in formula (3). Note that this is formulated as a curve following problem in the state space, i.e., here $h(x)$ is the identity mapping.

The more flexible the family of curves $\mathcal{G}$ is, the better approximation of the reachable set is possible to achieve.

Example 4 Consider the following system

$$
\begin{aligned}
& \dot{x}_{1}=-x_{1}+2 x_{2}-x_{2}^{2}+1, \\
& \dot{x}_{2}=-2 x_{2}+u
\end{aligned}
$$

where $-3 \leq u \leq 3$. We compute the set of states that can be reached from $(0,0)$ along straight lines

$$
x=g\left(s ; x^{1}\right)=\left(s x_{1}^{1}, s x_{2}^{1}\right)^{T}, s \in[0,1] .
$$




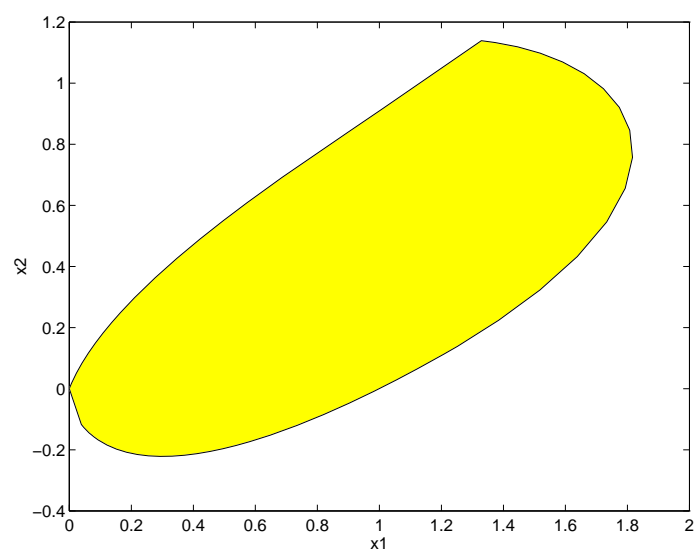

Figure 3: The set of states reachable along straight lines from the origin for system (10) using $-3 \leq u \leq 3$.

Quantifier elimination on a formula corresponding to (9) gives the set of states shown in Figure 3.

\section{Conclusions}

We have put the curve following problem, for dynamic systems subject to control constraints, in an algebraic framework. It has been shown how this problem can be formulated as a formula including quantifiers. We have used computer algebraic methods for quantifier elimination to synthesize admissible control laws. If the system description includes design parameters to be determined, the quantifier elimination procedure also gives necessary and sufficient conditions on these parameters for the formula to be true.

A slight generalization of the curve following problem gives approximations of reachable sets of systems subject to control constraints, which also has been treated in the paper.

\section{Acknowledgments}

This work was supported by the Swedish Research Council for Engineering Sciences (TFR), which is gratefully acknowledged. The author is also grateful to Dr. Hong at the Research Institute of Symbolic Computation (RISC), Austria for providing him with a copy of the QEPCAD program.

\section{References}

[1] C. T. Abdallah, P. Dorato, W. Yang, R. Liska, and S. Steinberg. Applications of quantifier elimination theory to control system design. In Proc. 4th IEEE Mediterranean Symp. on Control and Automation, 1996.
[2] B. Anderson, N. Bose, and E. Jury. Output feedback stabilization and related problems - solution via decision methods. IEEE Trans. Autom. Control, 1975.

[3] D.S. Arnon. A bibliography of quantifier elimination for real closed fields. J. Symbolic Comput., 5(12):267-274, 1988.

[4] G.E. Collins. Quantifier elimination for real closed fields by cylindrical algebraic decomposition. In $2 n d$ GI Conf. Automata Theory and Formal Languages. Springer, 1975.

[5] G.E. Collins and H. Hong. Partial cylindrical algebraic decomposition for quantifier elimination. $J$. Symbolic Comput., 1991.

[6] D. Cox, J. Little, and D. O'Shea. Ideals, Varieties, and Algorithms. Springer, 1992.

[7] J.H. Davenport, Y. Siret, and E. Tournier. Computer Algebra. Systems and Algorithms for Algebraic Computation. Academic Press, 1988.

[8] S. T. Glad. An algebraic approach to bang-bang control. In Proc. ECC'95, 1995.

[9] M. Jirstrand. Algebraic Methods for Modeling and Design in Control. Licentiate thesis LIU-TEKLIC-1996:05, Department of Electrical Engineering, Linköping University, Sweden, March 1996.

[10] M. Jirstrand and S. T. Glad. Computational questions of equilibrium calculation with application to nonlinear aircraft dynamics. In Proc. MTNS'96, St. Louis, USA, 1996.

[11] P. Lindskog. Methods, Algorithms and Tools for System Identification Based on Prior Knowledge. $\mathrm{PhD}$ thesis 436, Department of Electrical Engineering, Linköping University, Sweden, May 1996.

[12] B. Mishra. Algorithmic Algebra. Texts and Monographs in Computer Science. Springer-Verlag, 1993.

[13] D. Nesic and I. M. Y. Mareels. Deciding dead beat controllability using QEPCAD. In Proc. MTNS'96, St. Louis, USA, 1996.

[14] L.A. Rubel and M.F. Singer. A differentially algebraic elimination theorem with applications to ana$\log$ computability in the calculus of variations. In Proc.Amer.Math.Soc., volume 94, pages 653-658. Springer, 1985. 